\title{
Intrinsic organization of cortical networks predicts state anxiety: an functional near-infrared spectroscopy (fNIRS) study
}

\author{
Lian Duan', Nicholas T. Van Dam²,3, Hui Ai (i) ${ }^{1}$ and Pengfei Xu (i])
}

\begin{abstract}
Although state anxiety has been characterized by hyper-responsive subcortical activity and its bottom-up connectivity with cortical regions, the role of cortical networks in state anxiety is not yet well understood. To this end, we decoded individual state anxiety by using a machine-learning approach based on resting-state functional connectivity (RSFC) with functional near-infrared spectroscopy (fNIRS). Our results showed that the RSFC among a set of cortical networks were highly predictive of state anxiety, rather than trait anxiety. Specifically, these networks included connectivity between cortical areas in the default mode network (DMN) and dorsal attention network (DAN), and connectivity within the DMN, which were negatively correlated with state anxiety; connectivity between cortical areas in the DMN and frontoparietal network (FPN), FPN and salience network (SN), FPN and DAN, DMN and SN, which were positively correlated with state anxiety. These findings suggest a predictive role of intrinsic cortical organization in the assessment of state anxiety. The work provides new insights into potential neural mechanisms of emotion states and implications for prognosis, diagnosis, and treatment of affective disorders.
\end{abstract}

\section{Introduction}

Anxiety characterizes a subjective emotional state, relating to spatially or temporally distant and/or uncertain threat; it is often accompanied by autonomic arousal and behavioral avoidance $^{1,2}$. In contrast to fear which represents a more automated response to an imminent or immediate threat, anxiety is commonly associated with an individual's apprehension about potential or distant harm/threat ${ }^{2}$. While trait anxiety reflects an individual's predisposition for anxious responses, state anxiety reflects a temporary, subjective experience of apprehension about a potential threat or negative experience ${ }^{3,4}$. Thus, state anxiety represents a crucial situational relationship between physiological and

Correspondence: Hui Ai (h.ai@szu.edu.cn) or Pengfei Xu (pengfeixu.px@gmail. com)

${ }^{1}$ Shenzhen Key Laboratory of Affective and Social Neuroscience, Magnetic Resonance Imaging Center, Center for Brain Disorders and Cognitive Sciences, Shenzhen University, Shenzhen, China

${ }^{2}$ School of Psychological Sciences, University of Melbourne, Melbourne, Australia

Full list of author information is available at the end of the article subjective experiences of potential threat/harm ${ }^{5}$. State anxiety also reflects a potential precursor to broader tendencies in that the regular recurrence of particular states is likely to underpin the development of traits, over time ${ }^{6,7}$. While the veracity of subjective experience is, by definition, impossible to ascertain, objective markers of the subjective experience of fear may be critical to understanding why some people experience exaggerated threat responses while others do not $^{2}$. To be sure, these exaggerations likely stem from some critical vulnerabilities (e.g., elevated trait anxiety or neuroticism) but having some way of easily tracking the individual's state response as events are unfolding could hold much potential for predicting and treating anxiety disorders.

Although some studies suggest common brain networks among state, trait and pathological anxiety ${ }^{8,9}$, psychometric (and related brain imaging) analyses suggest that cognitive and somatic/physiological anxiety are quite different ${ }^{1,10,11}$. State anxiety has been shown to be more correlated with physiological/somatic symptoms than is trait anxiety ${ }^{12}$. Thus, state anxiety is more likely to reflect an individual's subjective

\section{(c) The Author(s) 2020}

(c) (i) Open Access This article is licensed under a Creative Commons Attribution 4.0 International License, which permits use, sharing, adaptation, distribution and reproduction cc) in any medium or format, as long as you give appropriate credit to the original author(s) and the source, provide a link to the Creative Commons license, and indicate if changes were made. The images or other third party material in this article are included in the article's Creative Commons license, unless indicated otherwise in a credit line to the material. If material is not included in the article's Creative Commons license and your intended use is not permitted by statutory regulation or exceeds the permitted use, you will need to obtain permission directly from the copyright holder. To view a copy of this license, visit http://creativecommons.org/licenses/by/4.0/. 
(bodily) attention to and/or interpretation of their present apprehension about imminent threat or harm, providing a key link between trait anxiety and the fear response. Indeed, recent work has shown that while trait anxiety tracks with intrinsic connectivity in midline cortical areas, state anxiety is more associated with activity in the insula ${ }^{13}$. Psychotherapeutic treatments for anxiety focus on reducing broader maladaptive behavioral patterns (consistent with trait anxiety) across treatment but also focus in session on experience at the moment, i.e., state anxiety; see, e.g., ref. ${ }^{14}$.

On the basis of neuroimaging studies for brain responses of anxious individuals to attentional ${ }^{15}$ and emotional ${ }^{16,17}$ processes, previous models highlight the role of hyperactive bottom-up input of the amygdala during specific processes in state anxiety ${ }^{18,19}$. However, recent a brain network model ${ }^{20}$ and meta-analysis ${ }^{9}$ have shown common connectivity patterns within and between various cortical areas between anxiety and anxiety disorders $^{21}$, including alterations in the frontoparietal control network (FPN), default mode network (DMN), dorsal attention network (DAN), ventral attention network, salience network (SN), and sensorimotor network. Interestingly, sustainedly altered activity in the vmPFC of the DMN has been observed during temporally persistent states of anxiety regardless of emotional manipulations ${ }^{22}$, pointing to alterations of intrinsic activity in state anxiety.

Resting-state functional connectivity (RSFC) is assumed to characterize commonly intrinsic representations of functional brain architecture across cognitive functions and is predictive of brain responses to task manipulations ${ }^{23}$ and individual difference ${ }^{24}$. While a recent proposed connectome-based predictive modeling model has been shown to effectively detect cognitive and brain state variability based on features from RSFC with full crossvalidation $^{25,26}$, both structural ${ }^{27}$ and functional ${ }^{28}$ connectivity of limbic areas with prefrontal regions have been shown to predict individual variations in trait anxiety. Individual differences in functional connectivity have been shown to be composed of both trait-relevant and state-dependent characteristics ${ }^{29}$. However, whether state anxiety could be predictable based on the intrinsic connectivity of brain networks, especially the cortical networks remain unclear. To this end, we conducted a functional near-infrared spectroscopy (fNIRS) study in individuals with various levels of state anxiety. We expected that the cortical areas in the previously proposed networks such as FPN and DMN should be able to predict state anxiety.

\section{Materials and methods \\ Participants and paradigm}

Ninety-six healthy young adults $(22.8 \pm 2.7$ years of age, 48 female) participated. The sample sizes were determined based on previous studies about the connectivity- based prediction of anxiety ${ }^{27,28}$. No participants had any history of psychiatric illness or neurological disease. All provided informed consent prior to the experiment. The study protocol was approved by the Institutional Review Board at Shenzhen University.

All participants underwent a 7-min session of restingstate fNIRS recording. They sat in a comfortable chair with their eyes closed and were instructed to keep still, to relax their mind, to remain awake, and not to think about anything systematically ${ }^{30}$. No participants reported sleep or were observed to be sleeping during fNIRS recording. After fNIRS data collection, participants completed the state and trait versions of the State-Trait Anxiety Inventory ${ }^{31}$.

\section{fNIRS data acquisition and preprocessing}

The fNIRS measurement was conducted with an ETG4000 continuous-wave optical topography system (Hitachi Medical Company, Tokyo, Japan). The absorption of the near-infrared light at two wavelengths $(695$ and $830 \mathrm{~nm})$ was measured with a sampling rate of $10 \mathrm{~Hz}$. Three pieces of probe sets were used, including one-piece placed on the frontal area and two pieces placed on the bilateral temporalparietal areas, forming 46 channels in total. The frontal probe set was placed by approximately putting its bottom middle optode on Fpz of the international 10-20 system ${ }^{32}$, and the bilateral temporal-parietal probe sets were placed by approximately putting their anterior inferior optode on T7 and T8, respectively (Fig. 1). The source-detector distance was $30 \mathrm{~mm}$. The cortical localizing MNI coordinates of the channels were obtained by using a 3-dimensional digitizer and the NIRS-SPM software ${ }^{33,34}$.

The light absorption data were converted to oxygenated $(\mathrm{HbO})$ and deoxygenated $(\mathrm{HbR})$ hemoglobin data via application of the modified Beer-Lambert law ${ }^{35}$ with a differential pathlength factor of $6.26^{36}$. The first and the last $10 \mathrm{~s}$ of data were discarded to ensure steady-state. Data were visually inspected to reject artifacts such as motion-related noises ${ }^{37,38}$. To remove superficial physiological noise and its related spurious connectivity ${ }^{39,40}$, the wavelet-based method was used to remove global physiological noise from the signal ${ }^{41}$. The data were band-pass filtered $(0.01-0.08 \mathrm{~Hz})$ to extract spontaneous neural activity ${ }^{42}$.

\section{Feature extraction}

To extract features for prediction of individual phenotypic differences, we defined the ANIRS channels as nodes within specific resting-state networks $\mathrm{cf}^{43}$. RSFC was calculated between each channel and all other channels, representing network edges, resulting in a resting-state network containing 46 nodes and 1035 edges for each participant. We defined the RSFC as the Pearson correlation coefficient between the time courses of each pair of channels ${ }^{44}$. All 1035 RSFC values made up the feature vector which was used to predict individual phenotypic variation. 


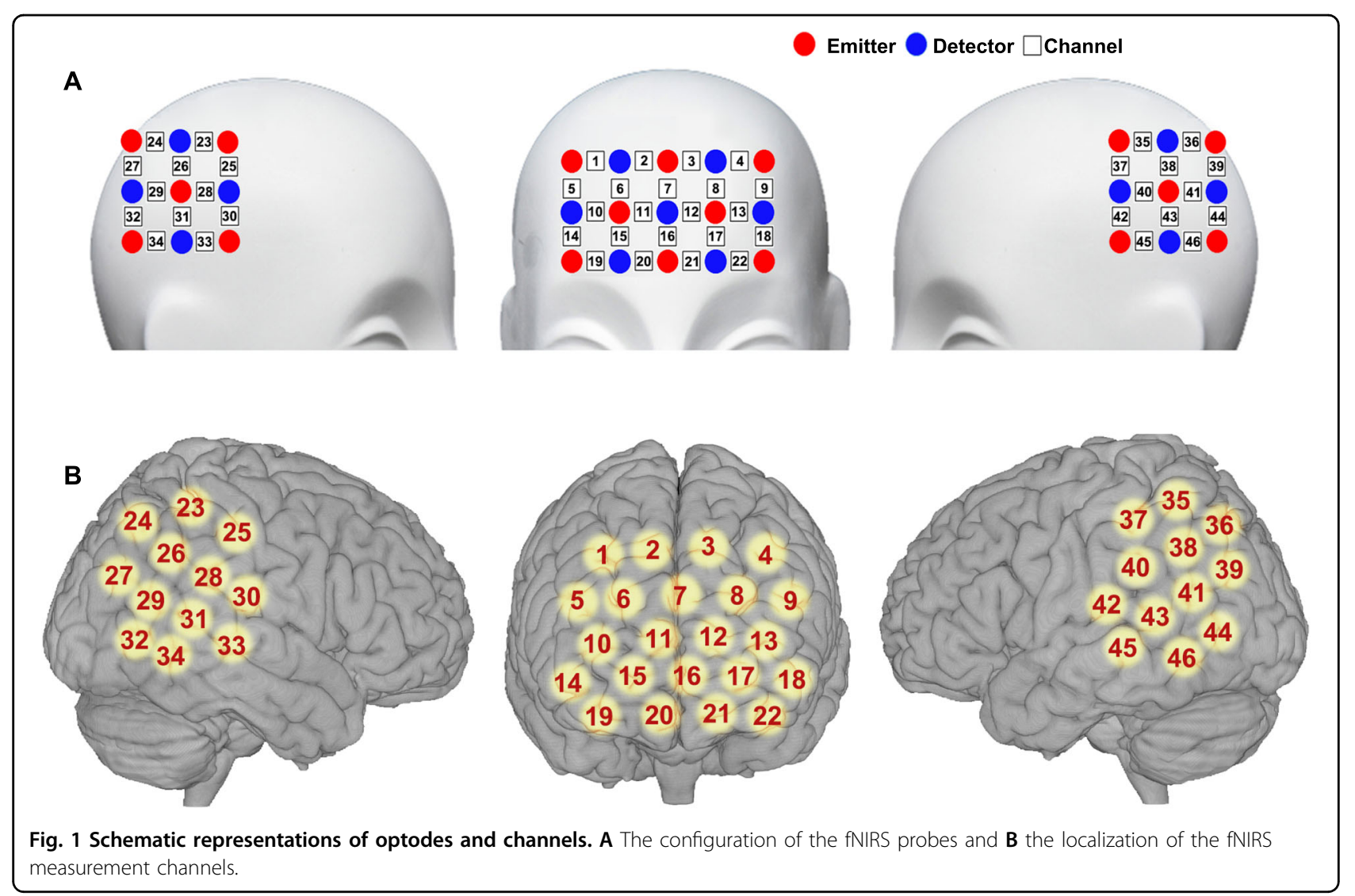

\section{Prediction model}

We built a multiple linear prediction models based on ridge regression (Fig. 2). Ridge regression is a regularized regression model which is particularly effective when the number of predictors is much bigger than the number of observations and collinearity exists among the predictors $^{45}$. To assess whether the regression model could predict the individual differences in anxiety, we used a stratified eightfold cross-validation approach with the nested cross-validation for the regularization parameter estimation $^{27}$. Specifically, we split the data of 96 participants eightfold. Each fold consisted of 12 participants and had a similarly distributed range of anxiety values. We performed eight iterations of model training and testing. In the $k$ th iteration, we kept the $k$ th fold of data as the testing set (12 samples) and used the remaining sevenfold of data as the training set (84 samples) to train the regression model. To determine the regularization parameter (alpha) of the ridge regression, for every iteration, we traversed the alpha values from 0.01 to 10 in steps of 0.01 . For every alpha value, we conducted a leave-one-out cross-validation (LOOCV) only using the training set. We averaged the alpha values corresponding to the maximum mean accuracy of the LOOCV across iteration to obtain a mean alpha value for model training. The mean alpha value was also used in a permutation test (see below). We tested the model of each iteration using the reserved testing set and produced 12 predicted anxiety scores. We concatenated the predicted anxiety scores from all the eight iterations and obtained a vector of 96 predicted anxiety scores, one predicted score for each participant. We calculated the accuracy of the predictions by computing the mean squared error (MSE) and the Pearson correlation coefficient $(r)$ between the predicted and actual state/trait anxiety scores.

To test the statistical significance of the accuracy of the predictions, we conducted a permutation test by randomly pairing the samples and the anxiety scores. We performed 10,000 permutations and calculated the MSE of the model fit. We determined the $p$ value as the proportion of iterations in which the MSE derived from the randomized data were smaller than or equal to that derived from the real data.

To validate the model performance, we also estimated the model by using different cross-validation schemes (4, $6,12,16$-fold), and calculated the correlation coefficients between actual and predicted anxiety scores.

\section{RSFC in the prediction of anxiety}

In order to determine which features (i.e., which resting-state functional connectivity) significantly contributed to the prediction of the individual differences in 


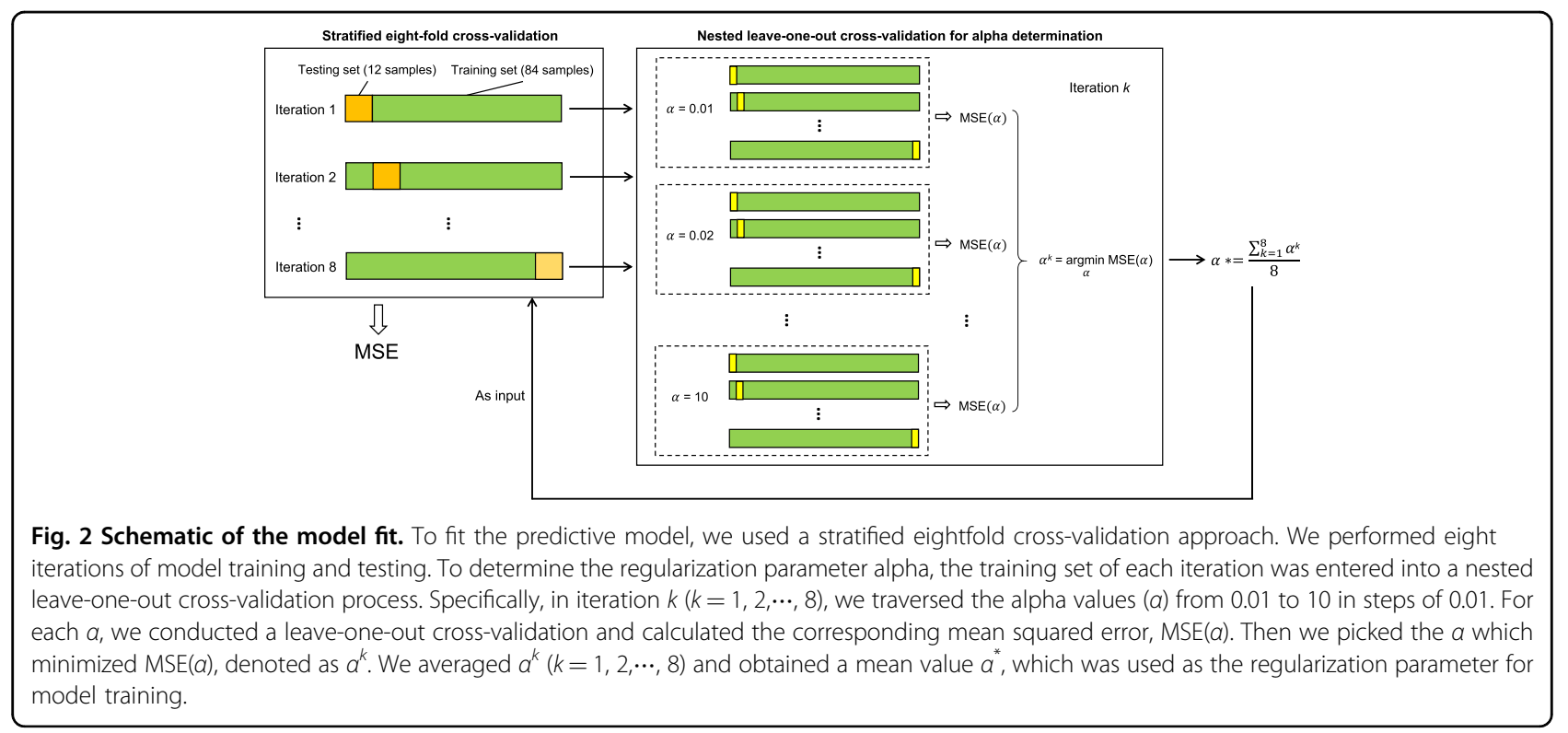

anxiety, we used a bootstrap approach as used in previous studies $^{27,46}$. Specifically, we performed 1000 bootstrap samplings with replacement. We generated 1000 independent regression models and estimated the 99\% confidence interval of each feature's weight. Those features whose $99 \%$ confidence interval was either entirely above or below zero were determined as significantly contributing features. To further characterize the connectivity properties of significant RSFC, we determined the anatomical localization and the resting-state network affiliation of terminal regions by using the automated anatomical labeling template ${ }^{47}$ and the seven-network brain surface parcellation template ${ }^{48}$, respectively.

\section{Results}

\section{Prediction model}

The models were used to attempt to predict both state and trait anxiety, separately. The scores of state anxiety ranged from 20 to 70 (Fig. 3A). The permutation tests showed that the ridge regression model could significantly predict levels of state anxiety above chance (mean alpha $=3.93, p<0.0054$, Fig. 3B). The MSE of our model was 122.04, while the mean MSE of the randomized permutation samples was 184.42. Pearson's correlation between the predicted state anxiety scores and self-reported state anxiety scores was significantly correlated $\left[r_{(95)}=0.36, p\right.$ $<3.43 \times 10^{-4}$, Fig. 3C]. The validation analysis of different cross-validation schemes showed that the results were very robust (Table 1$)$.

We also fitted the same model in trait anxiety (range: 22-66; Fig. 3D). The permutation test showed that the model was not significantly predictive of levels of trait anxiety $(p=0.474$, Fig. 3E). Predicted and self-reported trait anxiety were not significantly correlated $\left[r_{(95)}=0.03\right.$, $p=0.75$, Fig. 3F], though the state anxiety was highly correlated with those of trait anxiety $\left(r{ }_{(95)}=0.67, p<\right.$ $1.46 \times 10^{-13}$, Fig. $3 G$ ). To test whether the current predictive model is specific to the state rather than trait anxiety, we regressed state anxiety on trait anxiety. Then we used the connectivity-based model to predict the residuals. Results showed that the correlation between predictive scores and residuals was marginally significant $(r=0.20, p=0.051)$, suggesting that the current predictive model is largely associated with state anxiety, independent of trait anxiety.

\section{RSFC contributions to the prediction of state anxiety}

The bootstrap analysis determined the rsFC significantly contributed to the prediction model of state anxiety (Fig. 4; Fig. 5; Table 2). Generally, the contributing RSFC with negative weights was primarily distributed within DMN and between DMN and DAN (Fig. 4A, B; Fig. 5B), while the contributing RSFC with positive weights was primarily distributed between FPN and DMN, FPN, and DAN, as well as FPN and SN (Fig. 4C, D; Fig. 5C).

\section{Discussion}

In the current study, we established a connectomebased predictive model of state anxiety based on intrinsic connectivity between cortical networks, including DMN, FPN, DAN, and SN. These findings demonstrate a crucial role of cortical regions in the individualized prediction of state anxiety, extending the classical amygdala-centric model of state anxiety. The state anxiety specific predictive model reveals potential neural underpinnings for the distinction of state-trait anxiety. The prediction of state anxiety suggests prominent contributions of state- 


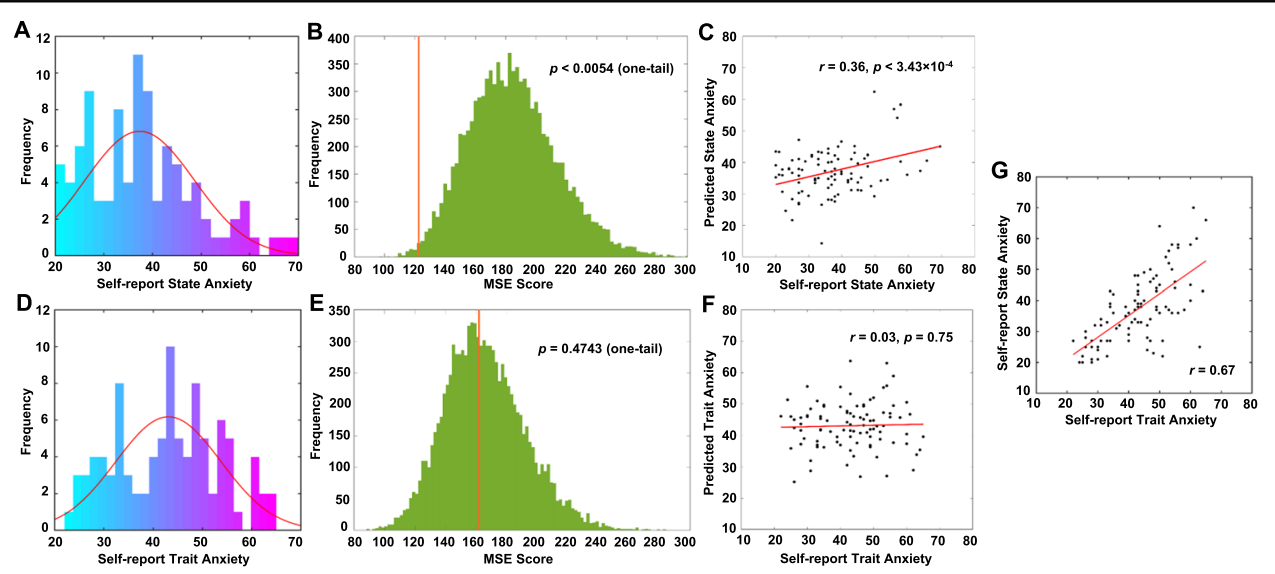

Fig. 3 Distribution of anxiety scores and performance of the prediction model. A Frequency distribution of levels of state anxiety. B Frequency distribution of the MSE scores for the prediction analysis in state anxiety. C Scatter plot for the relationship between self-report scores and predicted scores in state anxiety. D Frequency distribution of levels of trait anxiety. E Frequency distribution of the MSE scores for the prediction analysis in trait anxiety. F Scatter plot for the relationship between self-report scores and predicted scores in trait anxiety. G Scatter plot for the relationship between state and trait anxiety.

Table 1 Results of different fold number of crossvalidation.

\begin{tabular}{lllllll}
\hline & Alpha & \multicolumn{2}{l}{ MSE } & & & \multicolumn{1}{c}{} \\
\cline { 3 - 4 } \cline { 7 - 7 } & & MSE-value & $\boldsymbol{p}$ Value & & $\boldsymbol{r}$ Value & $\boldsymbol{p}$ Value \\
\hline 4-fold & 7.60 & 120.15 & 0.0076 & 0.35 & $4.26 \times 10^{-4}$ \\
6-fold & 5.58 & 122.75 & 0.0109 & 0.34 & $6.53 \times 10^{-4}$ \\
8-fold & 3.93 & 122.04 & 0.0054 & 0.36 & $3.43 \times 10^{-4}$ \\
12-fold & 3.42 & 124.55 & 0.0103 & 0.36 & $3.50 \times 10^{-4}$ \\
16-fold & 5.05 & 124.23 & 0.0101 & 0.35 & $3.97 \times 10^{-4}$ \\
\hline
\end{tabular}

dependent connectomic characteristics to the prediction of individual differences.

Our results reveal the intrinsic connectivity of cortical network contributes to the prediction of anxiety state. Although state anxiety has been characterized by hyperactive amygdala while trait anxiety is linked with hypoactive prefrontal top-down control in previous models ${ }^{18,19}$, abundant studies show cortical alterations in state anxiety as well. Increased intrinsic connectivity of the DMN with the insula of the SN has also been observed positively correlated with levels of state anxiety in both youth and adults ${ }^{49}$. Altered activation of the brain areas in the SN during threat monitoring has been linked with the individual differences in state anxiety ${ }^{50}$. State anxiety has also been shown to be associated with responses of the vmPFC to threat ${ }^{51}$. Alterations in cross-frequency coupling of cortical oscillations have also been observed to be associated with state anxiety ${ }^{52}$. While hyperactive amygdala representing hyper-functional stimulus-driven bottom-up processing, cortical alterations may indicate abnormal top-down response to the bottom-up input ${ }^{20}$. Hyperactivation in the amygdala in emotion processing in anxious patients has been shown to be associated with its decoupling with the DMN at rest, the strength of which was negatively correlated with state anxiety, suggesting a dysfunctional inhibition of the DMN on the subcortical amygdala activation in anxiety $^{53}$. Alterations of subcortical-cortical connectivity have been widely shown in both state and trait anxiety ${ }^{50}$ during task-specific cognitive processes $^{54,55}$ and task-nonspecific resting state ${ }^{56-58}$. Taken together, these brain features provide substantial candidates for the current predictive models, raising possibilities for the predictive role of cortical networks in anxiety states.

The model established in the current study is specific for the state rather than trait anxiety. State anxiety has been distinguished from trait anxiety by the definition of temporal and persistent characteristics of anxiety. In the psychometrical model, state anxiety is composed by cognitive worry and autonomic emotional dimensions, whereas trait anxiety consists of four dimensions including social evaluation, physical danger, ambiguous, and daily routines ${ }^{1}$. Although common brain networks between state and trait anxiety have been revealed by functional connectivity study ${ }^{8}$, differentiating neural mechanisms of transient versus sustained anxiety has also been shown in generating and regulating anxiety ${ }^{22}$. While a common neural pathway of the AI in the SN with amygdala has been shown between state and trait anxiety with the specificity that state and trait anxiety are associated with the functional and structural connectivity, respectively ${ }^{56}$. These findings converge to common and distinctive mechanisms between state and trait anxiety, providing a potential schemes for diagnosis and treatment of clinical syndromes of anxiety. 


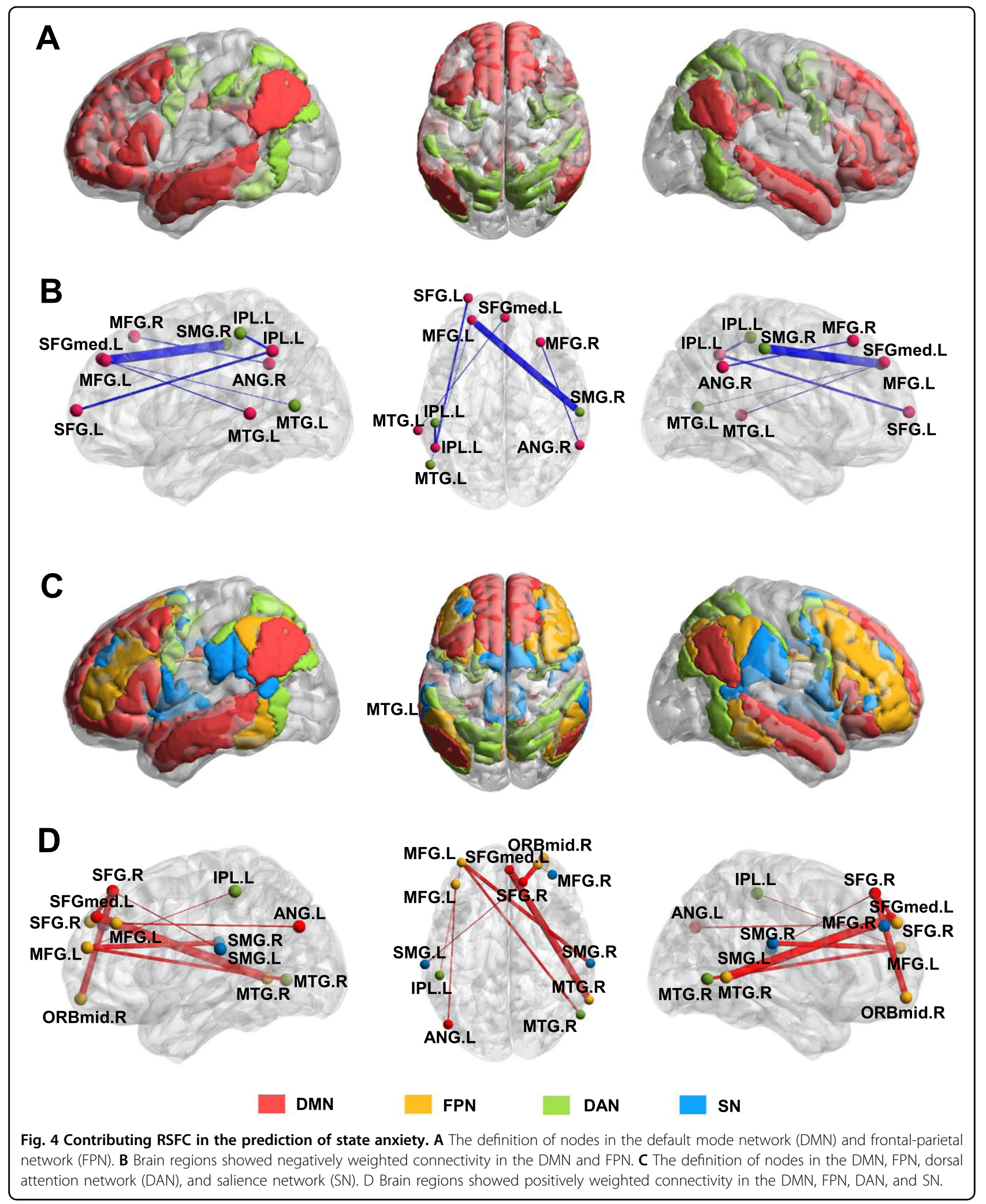

Our work provides evidence for prediction of emotion states, besides prediction of personality trait or psychopathology. The connectivity-based predictive model characterizes individual-specific trait profiles in a way as fingerprint ${ }^{59}$, which has been widely applied to cognitive functions ${ }^{24,26}$, personality trait ${ }^{28,60}$, and clinical 

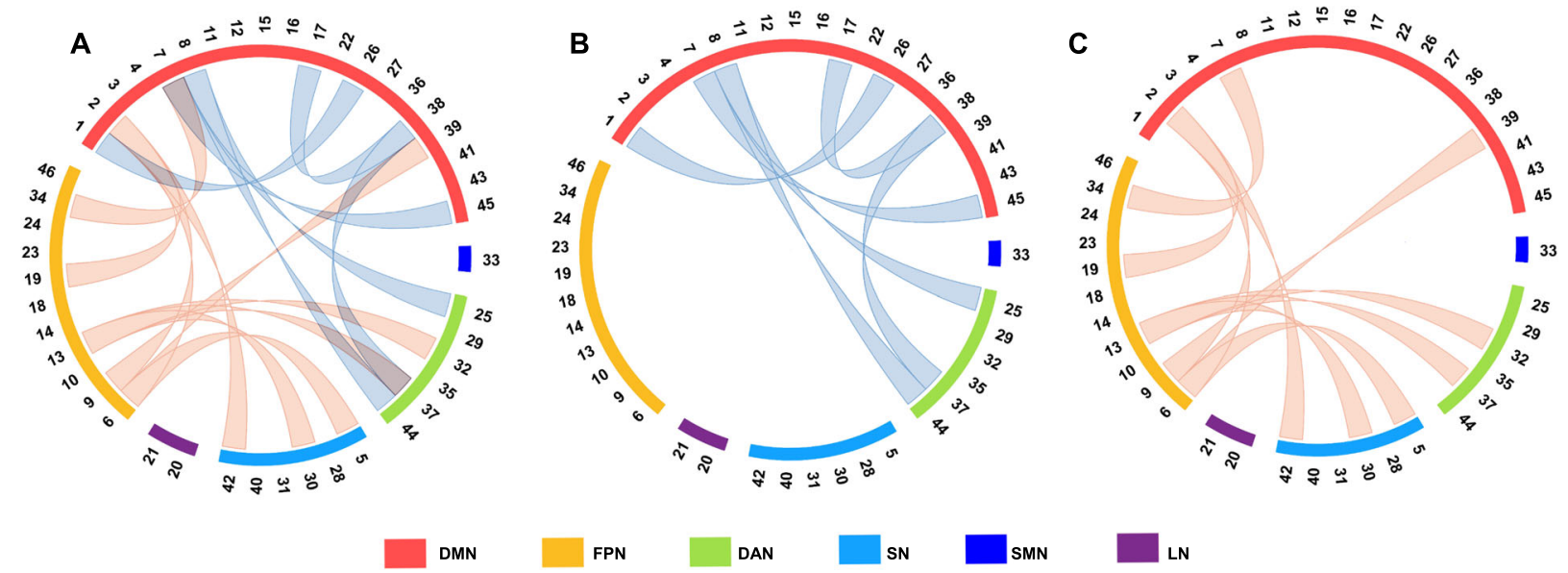

Fig. 5 Connectivity patterns of the six contributing networks. Patterns of $\mathbf{A}$ both negatively and positively weighted connectivity. B Connectivity within the DMN, between the DMN and DAN, negatively predicted levels of state anxiety. C Connectivity of the FPN with the DMN, DAN, and SN positively contributed to the prediction of state anxiety. See Fig. 1 for the location for each number of the optode. DMN default mode network, FPN frontoparietal network, DAN dorsal attention network, SN salience network, SMN somatomotor network, LN limbic network.

Table 2 Contributing RSFC in the prediction of state anxiety.

\begin{tabular}{|c|c|c|c|c|c|c|c|c|c|}
\hline \multirow[t]{2}{*}{ Feature (rsFC) } & \multicolumn{3}{|l|}{ Seed } & \multicolumn{3}{|l|}{ Target } & \multirow[t]{2}{*}{ Weight } & \multicolumn{2}{|c|}{ Confidence interval } \\
\hline & Channel & Region & Network & Channel & Region & Network & & & \\
\hline \multirow[t]{6}{*}{ Negative weight } & 1 & MFG.R & DMN & 26 & ANG.R & $\mathrm{DMN}$ & -0.6519 & -1.2696 & -0.0342 \\
\hline & 7 & SFGmed.L & DMN & 45 & MTG.L & $\mathrm{DMN}$ & -0.5978 & -1.1455 & -0.050 \\
\hline & 8 & MFG.L & DMN & 44 & MTG.L & DAN & -0.545 & -1.0374 & -0.0526 \\
\hline & 17 & SFG.L & DMN & 38 & IPL.L & DMN & -0.7883 & -1.5126 & -0.0639 \\
\hline & 8 & MFG.L & DMN & 25 & SMG.R & DAN & -1.6234 & -3.1666 & -0.0802 \\
\hline & 37 & IPL.L & DAN & 38 & IPL.L & $\mathrm{DMN}$ & -0.7641 & -1.514 & -0.0142 \\
\hline \multirow[t]{9}{*}{ Positive weight } & 2 & SFG.R & DMN & 6 & SFG.R & FPN & 0.8241 & 0.0626 & 1.5856 \\
\hline & 7 & SFGmed.L & DMN & 34 & MTG.R & FPN & 0.9947 & 0.0729 & 1.9165 \\
\hline & 13 & MFG.L & FPN & 30 & SMG.R & SN & 0.854 & 0.0615 & 1.6465 \\
\hline & 13 & MFG.L & FPN & 37 & IPL.L & DAN & 0.7145 & 0.0721 & 1.3569 \\
\hline & 5 & MFG.R & SN & 6 & SFG.R & FPN & 0.7113 & 0.0113 & 1.4114 \\
\hline & 2 & SFG.R & DMN & 19 & ORBmid.R & FPN & 0.9085 & 0.015 & 1.8019 \\
\hline & 2 & SFG.R & DMN & 42 & SMG.L & SN & 0.7256 & 0.043 & 1.4083 \\
\hline & 9 & MFG.L & FPN & 39 & ANG.L & DMN & 0.7408 & 0.0284 & 1.4532 \\
\hline & 13 & MFG.L & FPN & 32 & MTG.R & DAN & 0.8008 & 0.0005 & 1.6011 \\
\hline
\end{tabular}

$L$ left, $R$ right, MFG middle frontal gyrus, SFGmed superior frontal gyrus (medial part), IPL inferior parietal lobule, ANG angular gyrus, MTG middle temporal gyrus, SMG supramarginal gyrus, ORBmid middle frontal gyrus (orbital part), DMN default mode network, DAN dorsal attention network, SN salience network, FPN frontoparietal network.

disorders ${ }^{61}$. However, extensive evidence also shows indispensable contributions of state-dependent functional architecture to Individualized brain organization ${ }^{62,63}$. Brain state manipulations have been shown to improve the prediction of individual traits by amplifying individual variations in specific state ${ }^{64}$. State-independent cortical-subcortical dysconnectivity has been shown to be fundamental for psychosis prediction and characterization $^{65}$. These results jointly suggest that linking statedependent functional reorganization with an individual differences would contribute to the explanation of behavioral phenotypes and clinical symptoms. 
Potentials imitations of the current study should be noted. State anxiety in the current study could be more or less different from the task-evoked transient anxiety. What we measured was a self-reported temporal anxious state without any specific bottom-up input from task manipulations, which might be relatively tonic in comparison to transient anxiety response to a specific stimulus but relatively phasic compared to stable trait anxiety. Therefore, explanations of the state of anxiety should be cautious here. In addition, the measurement channels in the present study could not cover the whole brain because of limited fNIRS probes. However, the configuration in frontoparietal areas was priori defined in terms of the regions characteristic in anxiety ${ }^{20}$ and the regions with high connectivity variability that predominant in individualized prediction ${ }^{66}$. Last but not least, given the comorbidity of depression with anxiety, there might be potential influences of depression on the current results. Trait anxiety mitigated but did not eliminate, the associations of state anxiety with connectivity. While past work has shown that trait anxiety is associated with depression ${ }^{67}$, it is possible that depression had potential confounding effects on our findings. Given that the current study examined healthy individuals with various levels of state anxiety, future studies are necessary to test the utility of the current predictive model for clinical anxiety.

In conclusion, we demonstrate intrinsic connectivity between cortical networks is specifically predictive of individual anxiety state. These findings shed light on the prediction of emotional states and potential neural mechanisms of trait-state distinction in anxiety, which would have important implications for prognosis and diagnosis of anxiety and anxiety disorders.

\section{Acknowledgements}

This work was supported by the National Natural Science Foundation of China (31530031, 61503030, 31871137, 31700959, 31920103009, and 31671133), Young Elite Scientists Sponsorship Program by China Association for Science and Technology (YESS2018), Guangdong International Scientific Collaboration Project (2019A050510048), Guangdong Key Basic Research Grant (2018B030332001), Guangdong young Innovative Talent Project (2016KQNCX149), Guangdong Pearl River Talents Plan Innovative and Entrepreneurial Team grant (2016ZT06S220), Guangdong University Innovation Team Project (2015KCXTD009), Guangdong Basic and Applied Research Major Project (2016KZDXM009), Shenzhen-Hong Kong Institute of Brain Science-Shenzhen Fundamental Research Institutions (2019SHIBS0003), Shenzhen Science and Technology Research Funding Program (JCYJ20180305124819889, JCYJ20180507183500566, JCYJ20150729104249783, and CYJ20170412164413575) and Shenzhen Peacock Program (827-000235, KQTD2015033016104926).

\section{Author details}

'Shenzhen Key Laboratory of Affective and Social Neuroscience, Magnetic Resonance Imaging Center, Center for Brain Disorders and Cognitive Sciences, Shenzhen University, Shenzhen, China. ${ }^{2}$ School of Psychological Sciences, University of Melbourne, Melbourne, Australia. ${ }^{3}$ Department of Psychiatry, Icahn School of Medicine at Mount Sinai, New York, New York, USA. ${ }^{4}$ Beijing Key Laboratory of Applied Experimental Psychology, Faculty of Psychology, Beijing Normal University, Beijing, China. ${ }^{5}$ Center for Neuroimaging, Shenzhen Institute of Neuroscience, Shenzhen, China. ${ }^{6}$ Guangdong-Hong Kong-Macao Greater Bay Area Research Institute for Neuroscience and Neurotechnologies, Kwun Tong, Hong Kong, China
Conflict of interest

The authors declare that they have no conflict of interest.

\section{Publisher's note}

Springer Nature remains neutral with regard to jurisdictional claims in published maps and institutional affiliations.

Received: 8 June 2020 Revised: 4 October 2020 Accepted: 29 October 2020 Published online: 20 November 2020

\section{References}

1. Endler, N. S. \& Kocovski, N. L. State and trait anxiety revisited. J. Anxiety Disord. 15, 231-245 (2001)

2. LeDoux, J. E. \& Pine, D. S. Using neuroscience to help understand fear and anxiety: a two-system framework. Am. J. Psychiatry 173, 1083-1093 (2016).

3. Satpute, A. B., Mumford, J. A., Naliboff, B. D. \& Poldrack, R. A. Human anterior and posterior hippocampus respond distinctly to state and trait anxiety. Emotion 12, 58-68 (2012).

4. Spielberger, C. D., Gorsuch, R. L., Lushene, R., Vagg, P. R. \& Jacobs, G. A. Manual for the State-Trait Anxiety Inventory. (Consulting Psychologists Press, CA, 1970).

5. Pine, D. S. \& LeDoux, J. E. Elevating the role of subjective experience in the clinic: response to Fanselow and Pennington. Am. J. Psychiatry 174, 1120-1121 (2017).

6. Davidson, R. J. Affective neuroscience and psychophysiology: toward a synthesis. Psychophysiology 40, 655-665 (2003).

7. Pichon, S., Miendlarzewska, E. A., Eryilmaz, H. \& Vuilleumier, P. Cumulative activation during positive and negative events and state anxiety predicts subsequent inertia of amygdala reactivity. Soc. Cogn. Affect. Neurosci. 10 180-190 (2015).

8. Takagi, Y. et al. A common brain network among state, trait, and pathological anxiety from whole-brain functional connectivity. Neuroimage 172, 506-516 (2018)

9. $\mathrm{Xu}$, J. et al. Anxious brain networks: a coordinate-based activation likelihood estimation meta-analysis of resting-state functional connectivity studies in anxiety. Neurosci. Biobehav Rev. 96, 21-30 (2019).

10. Gros, D. F., Antony, M. M., Simms, L. J. \& McCabe, R. E. Psychometric properties of the State-Trait Inventory for Cognitive and Somatic Anxiety (STICSA): comparison to the State-Trait Anxiety Inventory (STAI). Psychol. Assess. 19, 369-381 (2007)

11. Bijsterbosch, J., Smith, S., Forster, S., John, O. P. \& Bishop, S. J. Resting state correlates of subdimensions of anxious affect. J. Cogn. Neurosci. 26, 914-926 (2014).

12. Kabacoff, R. I., Segal, D. L., Hersen, M. \& Van Hasselt, V. B. Psychometric properties and diagnostic utility of the Beck Anxiety Inventory and the StateTrait Anxiety Inventory with older adult psychiatric outpatients. J. Anxiety Disord. 11, 33-47 (1997)

13. Tian, $X$. et al. Assessment of trait anxiety and prediction of changes in state anxiety using functional brain imaging: a test-retest study. Neurolmage 133, 408-416 (2016).

14. Newmark, C. S. The effects of psychotherapeutic intervention on state and trait anxiety. J. Community Psychol. 2, 37-38 (1974).

15. Bishop, S. J., Duncan, J. \& Lawrence, A. D. State anxiety modulation of the amygdala response to unattended threat-related stimuli. J. Neurosci. 24, 10364-10368 (2004)

16. Somerville, L. H., Kim, H., Johnstone, T., Alexander, A. L. \& Whalen, P. J. Human amygdala responses during presentation of happy and neutral faces: correlations with state anxiety. Biol. Psychiatry 55, 897-903 (2004).

17. Etkin, A. et al. Individual differences in trait anxiety predict the response of the basolateral amygdala to unconsciously processed fearful faces. Neuron 44, 1043-1055 (2004)

18. Bishop, S. J. Neurocognitive mechanisms of anxiety: an integrative account. Trends Cogn. Sci. 11, 307-316 (2007).

19. Bishop, S. J. Neural mechanisms underlying selective attention to threat. Ann. N. Y Acad. Sci. 1129, 141-152 (2008)

20. Sylvester, C. M. et al. Functional network dysfunction in anxiety and anxiety disorders. Trends Neurosci. 35, 527-535 (2012).

21. Tovote, P., Fadok, J. P. \& Luthi, A. Neuronal circuits for fear and anxiety. Nat. Rev. Neurosci. 16, 317-331 (2015). 
22. Somerville, L. H. et al. Interactions between transient and sustained neural signals support the generation and regulation of anxious emotion. Cereb. Cortex 23, 49-60 (2013).

23. Mennes, M. et al. Inter-individual differences in resting-state functional connectivity predict task-induced BOLD activity. Neuroimage 50, 1690-1701 (2010).

24. Tavor, I. et al. Task-free MRI predicts individual differences in brain activity during task performance. Science 352, 216-220 (2016).

25. Shen, $X$. et al. Using connectome-based predictive modeling to predict individual behavior from brain connectivity. Nat. Protoc. 12 506-518 (2017)

26. Rosenberg, M. D. et al. A neuromarker of sustained attention from whole-brain functional connectivity. Nat. Neurosci. 19, 165-171 (2016).

27. Greening, S. G. \& Mitchell, D. G. A network of amygdala connections predict individual differences in trait anxiety. Hum. Brain Mapp. 36, 4819-4830 (2015).

28. Wang, Z. et al. Connectome-based predictive modeling of individual anxiety. bioRxiv https://doi.org/10.1101/2020.01.30.926980 (2020).

29. Geerligs, L., Rubinov, M., Cam, C. \& Henson, R. N. State and trait components of functional connectivity: individual differences vary with mental state. J. Neurosci. 35, 13949-13961 (2015)

30. Xu, P. et al. Different topological organization of human brain functional networks with eyes open versus eyes closed. Neuroimage 90 264-255 (2014).

31. Spielberger, C. D., Gorsuch, R. L., Lushene, R., Vagg, P. R., Jacobs, G. A. Manual for the State-Trait Anxiety Inventory. (Consulting Psychologist Press, Palo Alto, CA, 1983).

32. Jasper, H. H. The 10-20 Electrode System of the International Federation. Electroencephalography \& Clinical Neurophysiology 10, 371-375 (1958).

33. Singh, A. K., Okamoto, M., Dan, H., Jurcak, V. \& Dan, I. Spatial registration of multichannel multi-subject fNIRS data to MNI space without MRI. Neuroimage 27, 842-851 (2005).

34. Ye, J. C., Tak, S., Jang, K. E., Jung, J. \& Jang, J. NIRS-SPM: statistical parametric mapping for near-infrared spectroscopy. Neuroimage 44, 428 (2009).

35. Cope, M. \& Delpy, D. T. System for long-term measurement of cerebral blood and tissue oxygenation on newborn infants by near infra-red transillumination. Med. Biol. Eng. Comput. 26, 289-294 (1988).

36. Duncan, A. et al. Optical pathlength measurements on adult head, calf and forearm and the head of the newborn infant using phase resolved optical spectroscopy. Phys. Med. Biol. 40, 295-304 (1995).

37. Cooper, R. J. et al. A systematic comparison of motion artifact correction techniques for functional near-infrared spectroscopy. Front. Neurosci. 6, 147 (2012).

38. Pinti, P., Scholkmann, F., Hamilton, A., Burgess, P., \& Tachtsidis, I. Current status and issues regarding pre-processing of fNIRS neuroimaging data: an investigation of diverse signal filtering methods within a general linear model framework. Front. Hum. Neurosci. 12, 505 (2019).

39. White, B. R. et al. Resting-state functional connectivity in the human brain revealed with diffuse optical tomography. Neuroimage 47, 148 (2009).

40. Sakakibara, E. et al. Detection of resting state functional connectivity using partial correlation analysis: A study using multi-distance and whole-head probe near-infrared spectroscopy. Neuroimage 142, 590-601 (2016).

41. Duan, L. et al. Wavelet-based method for removing global physiological noise in functional near-infrared spectroscopy. Biomed. Opt. Express 9, 3805 (2018).

42. Biswal, B., Yetkin, F. Z., Haughton, V. M. \& Hyde, J. S. Functional connectivity in the motor cortex of resting human brain using echo-planar MRI. Magn. Reson. Med. 34, 537-541 (1995).

43. Power, J. D. et al. Functional network organization of the human brain. Neuron 72, 665-678 (2011).

44. Duan, L., Zhang, Y. J. \& Zhu, C. Z. Quantitative comparison of resting-state functional connectivity derived from fNIRS and fMRl: a simultaneous recording study. Neuroimage 60, 2008-2018 (2012).
45. Hoerl, A. E. \& Kennard, R. W. Ridge regression: biased estimation for nonorthogonal problems. Technometrics 12, 55-67 (1970).

46. Wager, T. D., Atlas, L. Y., Leotti, L. A. \& Rilling, J. K. Predicting individual differences in placebo analgesia: contributions of brain activity during anticipation and pain experience. J. Neurosci. 31, 439-452 (2011).

47. Tzourio-Mazoyer, N. et al. Automated anatomical labeling of activations in SPM using a macroscopic anatomical parcellation of the MNI MRI singlesubject brain. Neurolmage 15, 273-289 (2002).

48. Yeo, B. T. et al. The organization of the human cerebral cortex estimated by intrinsic functional connectivity. J. Neurophysiol. 106, 1125-1165 (2011).

49. Dennis, E. L., Gotlib, I. H., Thompson, P. M. \& Thomason, M. E. Anxiety modulates insula recruitment in resting-state functional magnetic resonance imaging in youth and adults. Brain Connect 1, 245-254 (2011).

50. Choi, J. M., Padmala, S. \& Pessoa, L. Impact of state anxiety on the interaction between threat monitoring and cognition. Neuroimage 59, 1912-1923 (2012).

51. Hu, K. Neural activity to threat in ventromedial prefrontal cortex correlates with individual differences in anxiety and reward processing. Neuropsychologia 117 566-573 (2018)

52. Knyazev, G. G. Cross-frequency coupling of brain oscillations: an impact of state anxiety. Int J. Psychophysiol. 80, 236-245 (2011).

53. Hahn, A. et al. Reduced resting-state functional connectivity between amygdala and orbitofrontal cortex in social anxiety disorder. Neuroimage 56, 881-889 (2011)

54. $\mathrm{Xu}, \mathrm{P}$. et al. Neural basis of emotional decision making in trait anxiety. J. Neurosci. 33, 18641-18653 (2013).

55. Fung, B. J., Qi, S., Hassabis, D., Daw, N., Mobbs, D. Slow escape decisions are swayed by trait anxiety. Nat. Hum. Behav. 3, 702-708 (2019).

56. Baur, V., Hanggi, J., Langer, N. \& Jancke, L. Resting-state functional and structural connectivity within an insula-amygdala route specifically index state and trait anxiety. Biol. Psychiatry 73, 85-92 (2013).

57. Vytal, K. E., Overstreet, C., Charney, D. R., Robinson, O. J. \& Grillon, C. Sustained anxiety increases amygdala-dorsomedial prefrontal coupling: a mechanism for maintaining an anxious state in healthy adults. J. Psychiatry Neurosci. 39 321-329 (2014).

58. Kim, M. J., Gee, D. G., Loucks, R. A., Davis, F. C. \& Whalen, P. J. Anxiety dissociates dorsal and ventral medial prefrontal cortex functional connectivity with the amygdala at rest. Cereb. Cortex 21, 1667-1673 (2011).

59. Finn, E. S. et al. Functional connectome fingerprinting: identifying individuals using patterns of brain connectivity. Nat. Neurosci. 18, 1664-1671 (2015).

60. Feng, C., Wang, L., Li, T. \& Xu, P. Connectome-based individualized prediction of loneliness. Soc. Cogn. Affect. Neurosci. 14, 353-365 (2019).

61. Wang, D. et al. Individual-specific functional connectivity markers track dimensional and categorical features of psychotic illness. Mol. Psychiatry 25 2119-2129 (2018)

62. Bolt, T., Nomi, J. S., Bainter, S. A., Cole, M. W. \& Uddin, L. Q. The situation or the person? Individual and task-evoked differences in BOLD activity. Hum. Brain Mapp. 40, 2943-2954 (2019)

63. Salehi, M., Karbasi, A., Barron, D. S., Scheinost, D. \& Constable, R. T. Individualized functional networks reconfigure with cognitive state. Neuroimage 206, 116233 (2020).

64. Greene, A. S., Gao, S., Scheinost, D. \& Constable, R. T. Task-induced brain state manipulation improves prediction of individual traits. Nat. Commun. 9, 2807 (2018).

65. Cao, H. et al. Cerebello-thalamo-cortical hyperconnectivity as a stateindependent functional neural signature for psychosis prediction and characterization. Nat. Commun. 9, 3836 (2018).

66. Mueller, S. et al. Individual variability in functional connectivity architecture of the human brain. Neuron 77, 586-595 (2013).

67. Bieling, P. J., Antony, M. M. \& Swinson, R. P. The State-Trait Anxiety Inventory, Trait version: structure and content re-examined. Behav. Res. Ther. 36, 777-788 (1998). 